\title{
Antioxidants, Oxidative Damage and Inflammation in Obese Subjects without Type-2 Diabetes Mellitus and Age and Gender Matched Obese Subjects with Type-2 Diabetes
}

\author{
Javed Yasin*, Awad El Essa, Juma Al Kaabi and Salah Gariballa \\ Department of Internal Medicine, United Arab Emirates University, United Arab Emirates
}

Submission: September 28, 2018; Published: November 21, 2018

*Corresponding author: Javed Yasin, Department of Internal Medicine, Faculty of Medicine and Health Sciences, United Arab Emirates University, PO Box 17666, Al-Ain, United Arab Emirates

Abstract

Background: The epidemic of visceral obesity and related type-2 diabetes in the United Arab Emirates (UAE) is continuing relentlessly. Possible mechanisms that link visceral obesity to diabetes include inflammation and increased oxidative stress; however, few data are available from the Arab population.

Methods: We compared baseline data of 48 obese subjects without type- 2 diabetes visiting primary health centers, with 48 age and sexmatched obese individuals with type 2 diabetes visiting the diabetes Centre. Outcome measures were lifestyle, anthropometric, antioxidants, oxidative damage and inflammatory markers.

Results: Mean (SD) age of each study group was 44 (9) years with 38 females and 10 males in each group. Obese subjects with diabetes have increased adiposity, high blood pressure (BP) and levels of physical inactivity compared with obese subjects without diabetes. After adjusting for glycemic control and medications inflammatory and oxidative damage markers were significantly higher in obese individuals with diabetes compared with the group without diabetes. No significant diffidence however found between the two groups in serum folate, vitamin A and $\mathrm{E}$ concentrations ( $p=0.072,0.137$, and 0.177 respectively). In contrast vitamin B12 levels were almost half in obese diabetic subjects [213 (105 $\mathrm{pg} / \mathrm{ml}]$ compared to obese subjects without diabetes $[410(162) \mathrm{pg} / \mathrm{ml}], \mathrm{p}<0.001$.

Conclusion: Obese diabetic subjects have increased adiposity, inflammation and oxidative damage. Markers of oxidative damage and inflammation may be used to identify and target obese subjects at high risk of complications in the UAE and similar populations.

Keywords: Obesity; Type-2 diabetes; Antioxidant; Oxidative damage; Inflammatory markers

Abbreviations: UAE: United Arab Emirates; BP: Blood Pressure; CVD: Cardiovascular Disease; BMI: Body Mass Index; ELISA: Enzyme-Linked Immunosorbent Assay;

\section{Introduction}

There is a growing epidemic of overweight/obesity, type-2 diabetes and other related cardiovascular disease (CVD) in The United Arab Emirates (UAE) [1-4]. As a result, the UAE has one of the highest prevalence of diabetes mellitus in the World [4]. Possible mechanisms that relate obesity to increased risk of diabetes include inflammation, oxidative damage, and related insulin resistance. In obese patients' subclinical inflammation has been found to correlate with markers of oxidative stress in adipose tissue and this may be the mechanism for obesityrelated metabolic syndrome, insulin resistance and diabetes mellitus [5]. Furthermore, both oxidative stress and low-grade inflammation may be causatively linked to the development, progression and complications of diabetes in obese patients [5-7]. Recent research reveals that oxidative stress occurs before the appearance of clinical manifestations of late diabetic complications, suggesting a key role in the pathogenesis of the disease [6]. Understanding some of these mechanisms that lead to the development of diabetes in obese subjects may help provide an important tool to help reduce the burden associated with obesity and related diabetes in the UAE society and similar populations. In search for this goal we compared obese subjects without diabetes with an age and sex matched obese individuals with type 2 diabetes accessing health care facilities in the UAE. 


\section{Current Research in Diabetes \& Obesity Journal}

\section{Methods}

Obese subjects without diabetes aged 18 years and above visiting primary health centres for assessment were approached and invited to take part in the study. An age and sex-matched obese individuals with type 2 diabetes mellitus visiting the diabetes centre at the Tawam hospital for follow up were recruited. The Tawam hospital and the primary health care in the city of Al Ain serve a total population of 400,000. Individuals with severe chronic clinical or psychiatric disease, participating in other intervention trials and those unable to give an informed written consent were excluded. Al Ain district research ethical committee has approved the study. Following informed written consent and their recruitment to the study, eligible subjects had demographic and medical data collected, including history of chronic diseases, drug intake, fruits and vegetables consumption, physical activity and a fasting blood sample taken for measurements of markers of inflammation and oxidative damage. Anthropometric data including body weight, height and body mass index (BMI) were measured using Tanita body composition analyser. Waist and hip circumference were measured using a flexible plastic tape.

A questionnaire was used to assess physical activity. Data were obtained on frequency and duration of daily or weekly physical activity sessions for at least 20 minutes or more in which subjects became breathless or sweating [8]. Measurement of fruits and vegetables intake was carried using an abbreviated semi-quantitative food frequency questionnaire designed for self-administration following a brief verbal discussion. It assesses the average weekly fruits and vegetables consumption of each individual. The full version of the questionnaire has been developed and validated against 7 day weighted dietary intake. It has also been compared with numerous other diet sand used in many other studies [9].

Results

Table 1: Comparison of lifestyle, anthropometric, blood pressure and lipid profile between type-2 obese diabetics and obese non-diabetic individuals (mean SD unless stated otherwise)

\begin{tabular}{|c|c|c|c|}
\hline & Obese Diabetics & Obese Non-Diabetics & P value \\
\hline Body weight $(\mathrm{kg})$ & $89.3(15)$ & $88.2(17)$ & 0.732 \\
\hline Body mass index & $35.9(6)$ & $34.9(6)$ & 0.364 \\
\hline Waist circumference $(\mathrm{cm})$ & $107(11)$ & $104(12)$ & 0.147 \\
\hline Hip circumference $(\mathrm{cm})$ & $118(11)$ & $115(11)$ & 0.196 \\
\hline Body fat $\%$ & $40.9(6)$ & $41.3(6)$ & 0.79 \\
\hline Fat free mass $\%$ & $51.2(7)$ & $47.6(6)$ & 0.022 \\
\hline $\begin{array}{l}\text { How often are you physically active for at least } 20 \text { minutes, where you become } \\
\text { out of breath and sweating* }\end{array}$ & $1.36(0.9)$ & $1.9(1.3)$ & 0.024 \\
\hline How physically active is your leisure time+ & $0.7(0.7)$ & $1.57(0.7)$ & 0.001 \\
\hline Systolic blood pressure $\mathrm{mm} \mathrm{Hg}$ & $130(15)$ & $121(14)$ & 0.007 \\
\hline Diastolic blood pressure $\mathrm{mm} \mathrm{Hg}$ & $79(10)$. & $73(8)$ & 0.002 \\
\hline Number taking statins & 35 & 2 & 0.001 \\
\hline Total cholesterol (mmol/L) & $4.68(1.1)$ & $5.01(0.9)$ & 0.219 \\
\hline HDL (mmol/L) & $1.034(0.2)$ & $1.022(0.2)$ & 0.85 \\
\hline
\end{tabular}

Fasting blood samples were drawn into 2 vacutainer tubes, containing potassium EDTA as anticoagulant. The samples were thoroughly mixed at room temperature and immediately transferred to the laboratory. Both tubes were centrifuged immediately for $10 \mathrm{~min}$ at 4000 rotations $/ \mathrm{min}$. Plasma and serum were collected and stored at $-80^{\circ} \mathrm{C}$ for future determinations of biochemical outcome measures. Antioxidants: Vitamins $\mathrm{E}$ and $\mathrm{A}$, analysis were carried out using HPLC, and it was performed on a Waters (Milford, MA) system gradient liquid chromatography (model515) with auto injector (model 717). For vitamin B12 and Folate analyses, we used reagent kit from access reagent kit from Beckman Coulter. We used commercially available enzymelinked immunosorbent assay (ELISA) methods to measure plasma TNF and IL6. Lipid Peroxidation: The concentration of the lipid peroxidation product TBARS was measured using an assay kit (no, 10009055) from Cayman Chemical Company, 1180 E. Ellsworth Rd., Ann Arbor, MI 48108. Protein Oxidation: The content of protein-bound carbonyls used to assess the extent of protein oxidation, was determined calorimetrically using reagent kit (10005020) from Cayman Chemical. Circulating levels of renal and liver functions, lipids and high sensitivity $\mathrm{C}$ reactive protein (hsCRP) were measured using an automated analyzer Integra 400 Plus (Roche Diagnostics, Mannheim, Germany).

All Statistical analyses were performed using SPSS version 21.0 (SPSS, Chicago, IL, US). Statistical comparisons between groups were carried out using univariate analysis of variance test. Differences between the two groups in blood pressure, oxidative damage and inflammatory markers were adjusted for glycemic control (HbA1C levels) and medications. P value $<0.05$ was considered significant.

\section{Blood samples}




\section{Current Research in Diabetes \& Obesity Journal}

\begin{tabular}{|c|c|c|c|}
\hline LDL (mmol/L) & $3.03(1)$ & $3.05(0.8)$ & 0.928 \\
\hline TG (mmol/L) & $1.59(1.7)$ & $1.073(0.4)$ & 0.138 \\
\hline HbA1C (\%) & $8.3(2.2)$ & $5.8(0.8)$ & 0 \\
\hline
\end{tabular}

${ }^{*}$ How often are you physically active for at least 20 minutes, where you become out of breath and sweating <1/week, 2=1-2/week, 3-4/week, 5-6/week, 7-8/week, >8/week. +How physically active is your leisure time: 1: Not very active; 2: Moderately active; 3: Very active; HDL: High Density Lipoprotein; LDL: Low Density Lipoprotein; TG: Triglycerides

Table 2: Comparison of antioxidant vitamins and markers of oxidative damage and inflammation between type-2 obese diabetics and obese non-diabetic individuals (mean SD unless stated otherwise).

\begin{tabular}{|c|c|c|c|}
\hline & Obese Diabetics & Obese Non-Diabetics & P value \\
\hline Vitamin_B12 $(\mathrm{pg} / \mathrm{ml})$ & $214(105)$ & $411(162)$ & 0 \\
\hline Vitamin A (ug/ml) ${ }^{*}$ & $0.62(0.3)$ & $0.46(0.3)$ & 0.137 \\
\hline IL6 $(\mathrm{pg} / \mathrm{ml}) \alpha$ & $3.33(2.2)$ & $3.02(2.5)$ & 0.582 \\
\hline TNF $\alpha(\mathrm{pg} / \mathrm{ml}) \alpha$ & $2.18(3)$ & $1.33(0.3)$ & 0.21 \\
\hline HsCRP $(\mathrm{mg} / \mathrm{L}) \alpha$ & $9.8(8)$ & $8.6(9)$ & 0.561 \\
\hline Protein Carbonyl $(\mathrm{nmol} / \mathrm{ml})+$ & $0.64(0.4)$ & $0.08(0.07)$ & 0 \\
\hline TBARS $(\mathrm{nmol} / \mathrm{ml})+$ & $11.68(12.1)$ & $3.06(1.4)$ & 0.001 \\
\hline
\end{tabular}

*Antioxidant; +markers of oxidative damage; a inflammatory markers; TBARS: Thiobarbituric Acid Reactive Substances.

A total of 48 obese subjects without diabetes and 48 age and gender matched obese subjects with type 2 diabetes mellitus were recruited to the study. Mean (SD) age of each study group was 44 (9) years with 38 females and 10 males in each group. Mean duration of diabetes was 2.1(0.9) years. Thirty-five individuals out of the 48 diabetics were on oral hypoglycemic tablets, 1 on insulin, 10 on both tablets and insulin and 2 controlled with diet treatment alone. Obese diabetic patients have increased adiposity measured by waist circumference, high BP and low levels of physical activity during work and leisure times compared with non-diabetic subjects (Table 1). Although there were some differences in lipid profile including total cholesterol, LDL, HDL and triglycerides, 35 obese diabetic patients were on lipid lowering medications compared to 2 subjects in the obese group without diabetes (Table 1). HbA1c concentrations were significantly higher in obese diabetic subjects as expected compared to non-diabetics individuals. Table 2 shows antioxidant vitamins, markers of oxidative damage and inflammation. In contrast to increased fruits and vegetables consumption, inflammatory and oxidative damage markers significantly increased in obese diabetic subjects compared with obese subjects without diabetes after adjusting for glycemic control and medications (Table 2).

Although antioxidant vitamins levels were higher in obese diabetic subjects compared to obese subjects without diabetes, differences between the two groups were not statistically significant after adjusting for fruits and vegetables consumption (Table 2). Similar differences in anthropometric and markers of inflammation and oxidative damage were found between female obese subjects with and those without diabetes diabetic with more pronounced difference in hsCRP [Obese females without diabetes=7.5 (6.8) $\mathrm{mg} / \mathrm{L}$ vs obese females with diabetes $=10.4$ (8.6), $\mathrm{p}=0.027$ ]. Vitamin B12 levels were almost half in obese diabetic subjects compared to obese subjects without diabetes (Table 2). We found no significant difference in vitamin B12 levels between diabetic patients taking metformin $(n=26)$ and those not taking metformin or those subjects on higher dose compared with those taking lower doses.

\section{Discussion}

We found increased adiposity, oxidative damage and inflammation in obese diabetic compared to obese individuals without diabetes independent of glycemic control and medications. This an important result given our recently reported finding of increased abdominal obesity in our population and that increased adiposity is associated with increased inflammation and decreased antioxidant status [10].

Possible mechanisms that relate obesity and diabetes to increased CVD risk include inflammation and oxidative damage. Recent research findings do support a close link between oxidative stress and diabetes evolution, revealing that oxidative stress occurs before the appearance of clinical manifestations of late diabetic complications, suggesting a key role in the pathogenesis of the disease [6]. Oxidative stress is defined as imbalance between the generation of free oxygen radicals and the antioxidant defense system and results from increased production of reactive oxygen species known to trigger cytotoxic reactions that are damaging to membrane lipids, proteins, nucleic acids and carbohydrates. A number of studies have reported an association between oxidative stress and insulin resistance and that some antioxidants may improve insulin resistance $[5,11]$.

Antioxidants found in fruit and vegetables known to promote health by combating oxidative stress and inflammation. Our study results also show increased fruits and vegetables consumption in obese diabetic subjects compared with obese subjects without diabetes which is almost certainly due to the dietary advice diabetic subjects get as part of their routine care. Consequently, antioxidant vitamins concentrations were non-significantly raised in obese diabetic subjects but not high enough to suppress oxidative damage and or inflammation, this 


\section{Current Research in Diabetes \& Obesity Journal}

could be due either dose or duration of rise in antioxidants in diabetic subjects. Nevertheless, a recent systematic review and meta-analysis has reported that increasing daily intake of green leafy vegetables could significantly reduce the risk of type 2 diabetes [12]. Another recent data also suggest that dietary antioxidants intake may be a predictor of the risk to develop metabolic syndrome features such as adiposity or impairments in systolic blood pressure, serum glucose and free fatty acids, and some inflammatory biomarkers in healthy subjects [13]. There are also preliminary reports of the beneficial role of higher fruits and vegetables intake in subjects with visceral obesity mediated by decreased inflammatory response, mitigation of oxidative damage associated with inflammatory cytokines that favor lipolysis and lipid oxidation instead of fat storage $[14,15]$.

Another important finding is the significantly lower vitamin B12 levels in diabetic patients compared to subjects without diabetes and the lack of association with metformin treatment which may be due to the small sample size. Both observation and randomized studies have yielded inconsistent results on relationship between metformin treatment and vitamin B12 reduction, but a recent systematic review found that metformin treatment induces vitamin B12 reduction and in a dose dependent manner [16]. Although no clinical studies have assessed the beneficial effects of vitamin B12 supplements in patients with type 2 diabetes or other patients taking metformin, it is reasonable to consider monitoring and giving supplement to those patients with low levels.

Although it is not possible to infer causal relationships from this cross-sectional report our findings if confirmed may have significant public health importance given increased prevalence of visceral obesity in our society and that increased adiposity is associated with increased inflammation and decreased antioxidant status10. Studies of the early mechanisms of oxidative damage and subclinical inflammation which link obesity and its complications including diabetes and the benefits of antioxidants found in fruit and vegetables known to promote health by combating oxidative stress and inflammation are urgently needed in this population to help reduce the burden of these diseases which have already reached epidemic proportions. In the meantime, inflammatory and oxidative damage biomarkers may be used to identify and target obese subjects at high risk of complications and perhaps monitor response to lifestyle modifications in the UAE and similar populations

\section{Funding}

This study was funded by the Emirates Foundation (grant no 049/2010).

\section{Ethical Approval}

All procedures performed in studies involving human participants were in accordance with the ethical standards of the institutional and/or national research committee and with the 1964 Helsinki declaration and its later amendments or comparable ethical standards.

\section{Informed Consent}

Informed consent was obtained from all individual participants included in the study.

\section{References}

1. (2000) Obesity: preventing and managing the global epidemic. Report of a WHO consultation, World Health Organ Tech Rep Ser 894. pp. 1-253.

2. McLellan F (2002) Obesity rising to alarming levels around the world. Lancet 359(9315): 1412.

3. SA Stanner, S James, R Foster (2005) Cardiovascular disease: Diet, Nutrition and Emerging Risk Factors, the launch of the BNF Task Force report. Nutrition Bulletin 30(4): 392-396.

4. Malik A, Babir A, Abi Saab B, Roglic G, King H (2005) Glucose intolerance and associated factors in the UAE. Diabetes Research \& Clinical Practice 69: 188-195.

5. Evans J, Goldfine ID, Maddux BA, Grodsky GM (2002) Oxidative stress and stress activated Signalling pathways: A unifying hypothesis of Type 2 Diabetes. Endocrine Reviews 23(5): 599-622.

6. Hill MF (2008) Emerging role for antioxidant therapy in protection against diabetic cardiac complications: Experimental and clinical evidence for utilization of classic and new antioxidants. Current Cardiligy Reviews 4(4): 259-268.

7. Feng J He, Caryl A Nowson, Graham A MacGregor (2006) Fruit and vegetable consumption and stroke: meta-analysis of cohort studies. Lancet 367(9507): 320-326.

8. Gariballa S, Afandi B, Abu Haltem M, Yassin J, Alessa A (2013) Effect of antioxidants and B-group vitamins on risk of infections in patients with type 2 diabetes mellitus. Nutrients 5(3): 711-724.

9. Yarnell JW, Fehily AM, Millbank JE, Sweetnam PM, Walker CL (1983) A short dietary questionnaire for use in epidemiological survey: comparison with weighed dietary records. Hum Nutr Appl Nutr 37(2): 103-112.

10. Gariballa S, Kosanovic M, Yasin J, Essa AE (2014) Oxidative damage and inflammation in obese diabetic Emirati subjects. Nutrients 6(11): $4872-4880$.

11. Evans JL (2007) Antioxidants: do they have a role in the treatment of insulin resistance? Indian J Med Res 125(3): 355-372.

12. Carter P, Gray LJ, Troughton J, Khunti K, Davies MJ (2010) Fruit and vegetable intake and incidence of type 2 diabetes mellitus: systematic review and meta-analysis. BMJ 341: c4229.

13. Puchau B, Zulet A, Gonzalez A, Hermsdorff H, Martinez J (2010) Dietary total antioxidant capacity is negatively associated with some metabolic syndrome features in healthy young men. Nutrition 26(5): 534-541.

14. Katcher HI, Legro RS, Kunselman AR, Gillies PJ, Demers LM, et al. (2008) The effects of a whole grain enriched hypocaloric diet on cardiovascular disease risk factors in men and women with metabolic syndrome. Am J Clin Nutr 87(1): 79-90.

15. Koh-Banerjee P, Rimm EB (2003) Whole grain consumption and weight gain: a review of the epidemiological evidence, potential mechanisms and opportunities for future research. Proc Nutr Soc 62(1): 25-29.

16. Liu Q Li S, Quan H, Li J (2014) Vitamin B12 status in metformin treated patients: Systematic review. PLos One 9(6): e100379. 


\section{Current Research in Diabetes \& Obesity Journal}

(C) This work is licensed under Creative

OI: 10.19080/CRDOJ.2018.09.555757

Your next submission with Juniper Publishers will reach you the below assets

- Quality Editorial service

- Swift Peer Review

- Reprints availability

- E-prints Service

- Manuscript Podcast for convenient understanding

- Global attainment for your research

- Manuscript accessibility in different formats

( Pdf, E-pub, Full Text, Audio)

- Unceasing customer service

Track the below URL for one-step submission https://juniperpublishers.com/online-submission.php 